\title{
COMPACT GROUPS OF GALAXIES
}

\author{
P. HICKSON \\ University of British Columbia, Dept. Physics \& Astronomy \\ 2219 Main Mall, Vancouver, B.C. V6T1Z4 Canada
}

\begin{abstract}
This paper reviews some of the outstanding questions concerning compact groups of galaxies. These relate to the physical nature and dynamical status of the groups, their formation and evolution, and their role in galaxy evolution. The picture that emerges is that compact groups are generally physically dense systems, although often contaminated by optical projections. Their evolution is likely a continuous process of infall, interaction and merging. As new galaxies are added, and previous ones merge, the membership of the group evolves. I suggest that while the size of the group changes little, other physical properties such as total mass, gas mass, velocity dispersion, fraction of early-type galaxies increase with time. This picture is at least qualitatively consistent with observations and provides a natural explanation for the strongest correlations found in compact group samples.
\end{abstract}

\section{Introduction}

One hundred and twenty years have passed since the discovery of the first compact group, Stephan's celebrated Quintet [41]. We now know of several hundred small groups of galaxies which have similar properties. They contain typically four or five galaxies in close proximity in the sky. With apparent space densities as high as $10^{6} \mathrm{Mpc}^{-3}$, they are the densest known galactic systems. From the start, these systems have been enigmatic. When the first redshifts were obtained for galaxies in Stephan's Quintet, Seyfert's Sextet and VV 172, a remarkable chain of five galaxies, difficulties arose. All of these systems contain a galaxy whose redshift is discordant [5] [6] [40]. Even if the discordant redshifts are ignored, the velocity dispersion of the remaining galaxies is larger than that expected from the visible mass [5]. This discrepancy is now attributed to the presence of dark matter permeating the group and dominating its dynamics. Later, however, it became apparent that, even if the groups are dynamically bound, they would be 
unstable. Orbital decay due to dynamical friction should lead to stripping of galaxy halos, and eventual mergers, on relatively short time-scales [18] [7]. One must then explain how compact groups continue to form, and identify their progenitors and progeny. This leads to the larger question of the role of compact groups in galaxy evolution in general.

These difficulties would be alleviated if the groups themselves are not physically dense, and several alternative explanations for their high apparent density have been suggested. The question can in principle be settled by observations, and much progress in this area has been made over the past 15 years. It is timely to now ask what has been learned, and what is the status of the main questions regarding compact groups. The definition and selection of compact groups, and the main observational and theoretical results have recently reviewed by Hickson [14]. This paper briefly summarizes the current situation with regard to the most outstanding issues.

\section{Physical Nature}

It is generally accepted that the discordant redshifts seen in many compact groups are cosmological, the result of chance alignments of unrelated background or foreground galaxies. Statistical analysis (eg. [23]) suggests that the surprisingly large fraction of groups containing discordant redshifts (half of all quintets!) is consistent with chance alignments, although the subject continues to cause debate [1] [43].

Turning now to the accordant galaxies, we find several hypotheses that explain the apparent high space densities in compact groups:

Dense bound configurations. The natural explanation is that compact groups are indeed physically dense. Even after allowing for the modest increase in apparent density caused by selection biases [14], the typical physical separation between galaxies is only of order $50 h^{-1} \mathrm{kpc}$. This hypothesis is supported by a variety of observational evidence [19]. Foremost is the high fraction of interactions seen both in the morphology and kinematics of compact group galaxies [37] [27]. More recently, strong evidence for this hypothesis has come from the detection of diffuse X-rays from a large fraction of compact groups (eg. [33]).

Transient configurations. Rose [38] first suggested that compact groups may be transient configurations caused by the accidental coincidence of galaxies passing unusually close to each other in their orbits. While otherwise attractive, the hypothesis is statistically improbable as the chance of four or more galaxies momentarily occupying the same small volume is very low [19].

Alignments within loose groups. Long championed by Mamon [25], the hypothesis remains a viable explanation for at least some compact groups. 
The presence of at most two interacting galaxies may be explained by including a physical binary. However, the hypothesis fails to account for the many groups which contain more than two interacting galaxies or extended $\mathrm{X}$-ray emission.

Alignments within filaments. Hernquist et al. [12], have recently suggested that compact groups may arise as projections along filaments. Their numerical simulations indicate that motions of widely-separated galaxies in filaments can conspire to produce apparent velocity dispersions comparable to those seen in compact groups. It offers an explanation of the relatively low X-ray luminosities of most compact groups, but appears now to be inconsistent with observations [33].

\section{Relation to Environment}

From the preceding discussion, we are faced with the probability that many, perhaps most, compact groups are dense bound systems. It is then natural to ask how they relate to their environment. Although many compact groups appear to be quite isolated, they generally trace the overall galaxy distribution, and are found in low-density associations, filaments, and loose groups. The fact that few compact groups are found in rich clusters is in part due to the selection criteria employed (eg. [13]), but dynamical disruption of small groups during cluster collapse may also play a role. There are, however, a number of important differences between galaxies in compact groups and those in their environment. For example, the fraction of elliptical galaxies is significantly higher in compact groups than in the field and correlates with velocity dispersion [16].

Several authors have investigated the formation of compact systems by gravitational collapse within loose groups (eg [9]). This seems to be a plausible mechanism, although it remains to be seen whether or not the timescales are consistent with the properties of the neighborhoods of observed compact groups [43].

If compact groups condense from loose groups, they appear to have lost all dynamical memory of their origins. Neither the shapes and orientations of the groups, nor the orientation of the galaxy spin axes, correlate with the distribution or orientation of surrounding galaxies [32].

\section{Interactions}

Because of their high density and relatively low velocity dispersions (next section), interactions in compact groups should be both frequent and strong. As expected, there are many cases of strong interactions leading to star formation and possible mergers in these systems [36] [24] [34] [28] [35] [45] [31] [46] [21] [22]. While the level of star formation in compact groups is en- 
hanced with respect to that of isolated galaxies, it is lower than that found in strongly-interacting galaxy pairs [30] [44].

An interesting question is how interactions affect the environment of the galactic nuclei. Do they trigger nuclear star formation and/or nuclear (AGN) activity? A study of the nuclear regions of compact-group spiral galaxies indicates an order-of-magnitude enhancement in the star-formation rates compared to isolated spiral galaxies [29]. From spectra of 67 galaxies in compact groups, Coziol et al. [8] find $22 \%$ are starburst and $40 \%$ are (mostly low luminosity) AGN. The probability of finding an AGN is higher in luminous galaxies and early-type galaxies.

At higher luminosities, there is much circumstantial evidence that nuclear activity may be triggered by interactions in small groups of galaxies. A large fraction of ultraluminous infrared galaxies contain Seyfert nuclei, and essentially all are interacting [39]. Many QSOs appear to be interacting with close companions (eg. [42] [20] [3]). These may be extreme examples of interactions in compact groups, not found in local samples because of their rarity.

\section{Dynamical Status}

The observed velocity dispersions of compact groups range from a few tens to several hundreds of $\mathrm{km} \mathrm{sec}^{-1}$, with a median value of order $200 \mathrm{~km}$ $\sec ^{-1}$. When combined with their small sizes, this gives crossing times $t_{\mathrm{c}}$ which range from $10^{-3}$ to $\sim 1$ Hubble time, the median being $0.02 \mathrm{H}^{-1}$. Galaxies lose energy when moving through a background of lower-mass dark matter particles. The resulting dynamical friction timescale $t_{\mathrm{d}}$ depends on the amount and distribution of the dark matter, but for typical groups is of order $10 t_{c}$. One expects orbital decay and merging to occur on this timescale, as is confirmed by dynamical simulations which indicate that groups typically evolve to form a single massive remnant, resembling an elliptical galaxy, within a few crossing times [4].

As it is unlikely that the present epoch is special this process has likely continued for of order a Hubble time, so one is led to the conclusion that the population of remnants could exceed the current population of compact groups by an order of magnitude. Since current estimates place the luminosity density of present-day compact groups at a few percent of the luminosity density of the universe, it follows that compact-group remnants should comprise a substantial fraction of all galaxies. While this may not at first seem implausible, it is not at all clear that the properties of such remnants are consistent with those of elliptical galaxies, or of any other identifiable population [44]. Just considering the luminosities, a difficulty arises because the total luminosity of individual compact groups exceeds 
that of typical elliptical galaxies by several magnitudes [26].

The problem may be avoided if individual compact groups can survive for a Hubble time or more. Recent numerical results indicate that it is possible for groups to survive this long given either special initial conditions [10] or massive dark matter halos [2]. Alternatively, groups may be replenished by the addition of new galaxies. Governato et al [11] point out that in a high-density $(\Omega \simeq 1$ ) universe, infall of surrounding galaxies onto a group can rejuvenate the system, prolonging its life. This reasonable picture may have observational support. Moles et al [31] present evidence for an example of recent infall in Stephan's Quintet.

\section{Evolution}

It is interesting to explore the logical consequences of the infall picture, and to see if it leads to testable predictions. Suppose, for example, that compact groups result from a continuous process of infall, interaction and merging. Assume that the infalling galaxies are gas-rich, and that some fraction of the interactions and mergers lead to elliptical and S0 galaxies. At each stage in the process we apply the compact group selection criteria to define the group members. One finds [15] that the membership of the group changes with time, as galaxies merge, and as new galaxies join the group. The radius of the group, however, remains roughly constant - it is determined more by the selection criteria than by any intrinsic size of the group.

In this simple model we find that, for any individual group, the following parameters increase with time:

- mass - grows as galaxies fall into the group.

- velocity dispersion - increases with mass.

- elliptical and S0 fraction - grows due to the effect of interactions and mergers

- halo mass - grows as individual galaxy halos are disrupted

- diffuse gas mass - grows due to stripping of galactic gas

- X-ray luminosity - increases with gas mass

- X-ray temperature - increases with velocity dispersion

The rate at which this evolution occurs depends on the initial density contrast of the perturbation which gives rise to the group. As this is likely to have a wide range of possible values, we expect to find groups in various stages of dynamical evolution. Those groups with the highest fraction of elliptical galaxies would be the most evolved.

From this picture we can infer the following observable consequences:

- Physical properties should not correlate strongly with group radius

- Spiral fraction should correlate inversely with velocity dispersion 
- X-ray luminosity and temperature should correlate inversely with spiral fraction

- The mass and luminosity of the largest elliptical galaxy should correlate with velocity dispersion

- We should find a wide range of ages of stellar populations within compact group galaxies

- If globular clusters and/or dwarf galaxies are produced in interactions and mergers, the specific frequencies of these objects should correlate with group velocity dispersion

Comparing with observational results, we find that indeed, compact group parameters do not correlate strongly with radius. The morphology-density relation is absent or weak, velocity dispersion does not correlate with radius, etc. In contrast, the strong inverse correlation between spiral fraction and velocity dispersion is nicely explained. Looking at the X-ray results, we see that the relatively weak emission seen from spiral rich groups can result from the earlier evolutionary phase of these groups. An examination of the data reported by Hickson et al. [17] reveals that the luminosity of the brightest elliptical galaxy does indeed correlate with velocity dispersion, as predicted. The last two predictions have not yet been tested, but can in principle be determined by future observations.

In summary, we find that a self-consistent picture of the formation and evolution of compact groups emerges which is at least qualitatively in agreement with current observational data. Moreover, predictions are made which are amenable to observational tests.

In conclusion, compact groups have emerged as mostly dense physical systems, although contamination by optical projections is common. They are centres for gravitational interaction and the dynamical evolution of galaxies. They are by no means rare objects, but involve several percent of the total galactic population. Their physical properties, which cover a large range of values, are a function of the selection criteria by which they are identified, as would be expected if they represent the high-density tail of the clustering hierarchy.

\section{References}

1. Arp C, 1997, ApJ, 474, 74

2. Athanassoula E, Makino J \& Bosma A, 1997, MNRAS, 286, 825

3. Bahcall JN, Kirharos S, Saxe DH \& Schneider DP, 1997, ApJ, 479, 642

4. Barnes JE, 1989, Nature, 338, 123

5. Burbidge EM, \& Burbidge GR, 1959, ApJ, 130, 23

6. Burbidge EM, \& Burbidge GR, 1961, ApJ, 134, 244

7. Carnevali P, Cavaliere A \& Santangelo P, 1981, ApJ, 249, 449

8. Coziol R, Ribeiro ALB, de Carvalho RR \& Capelato, 1997, preprint

9. Diafario A, Geller MJ \& Ramella M, 1994, AAJ, 107, 868 
10. Governato F, Chincarini G, Bhatia R, 1991, ApJ, 371, L15

11. Governato F, Tozzi P, Cavaliere A, 1996, ApJ, 458, 18

12. Hernquist L, Katz N, Weinberg DH, 1995, ApJ, 442, 57

13. Hickson $\mathrm{P}, 1982, \mathrm{ApJ}, 255,382$

14. Hickson P, 1997, ARAA, 35, 357

15. Hickson $\mathrm{P}, 1998$, in preparation

16. Hickson P, Kindl E \& Huchra JP, 1988, ApJ, 331, 64

17. Hickson P, Mendes de Oliveira C, Huchra JP \& Palumbo GGC, 1992, ApJ, 399, 353

18. Hickson P, Richstone DO \& Turner EL, 1977, ApJ, 213, 323

19. Hickson P \& Rood HJ, 1988, ApJ, 331, L69

20. Hutchings JB, 1995, AJ, 109, 928

21. Iglesias-Paramo J, Vilchez JM, 1997, ApJ, 479, 190

22. Iglesias-Paramo J, Vilchez JM, 1997, ApJ, 489, L13

23. Iovino A \& Hickson P, 1997, MNRAS, 287, 21

24. Longo $\mathrm{G}$, et al, 1994, A\&Ap, 281, 418

25. Mamon GA, 1986, ApJ, 307, 426

26. Mendes de Oliveira C \& Hickson P, 1991, ApJ, 380, 30

27. Mendes de Oliveira C \& Hickson P, 1994, ApJ, 427, 684

28. Menon TK, 1995, AJ, 110, 2605

29. Menon TK, 1995, MNRAS, 274. 845

30. Moles M, et al, 1994, A\&Ap, 285, 404

31. Moles M, Sulentic JW \& Márquez I, 1997, ApJ, 485, L69

32. Palumbo GGC, et al., 1993, ApJ, 405, 413

33. Ponman TJ, Bourner PDJ, Ebeling H, \& Bohringer H, 1996, MNRAS, 283, 690

34. Rodrigue M, et al, 1995, AJ, 109, 2362

35. Ribeiro ALB, et al, 1996, ApJ, 463, L5

36. Rubin VC, Ford WKJ \& Hunter DA, 1990, ApJ, 365, 86

37. Rubin VC, Hunter DA \& Ford WKJ, 1991, ApJ Suppl, 76, 153

38. Rose JA, 1977, ApJ, 211, 311

39. Sanders DB \& Mirabel IF, 1996, ARAA, 34, 749

40. Sargent WLW, 1968, ApJ Lett, 153, L135

41. Stephan ME, 1877, MNRAS, 37, 334

42. Stockton A, 1982, ApJ, 257, 33

43. Sulentic JW, 1997, ApJ, 482, 640

44. Sulentic JW \& Raba ca 1994, ApJ, 429, 531

45. Verdes-Montenegro L et al., 1997, A\&Ap, 321, 409

46. Yen MS, Verdes-Montenegro L, del Olmo A \& Perea J, 1997, ApJ, 475, L21 